\title{
NÍVEIS DE INCLUSÃO DA TORTA DA SEMENTE DE CUPUAÇU NA DIETA DE FRANGOS CAIPIRAS CRIADOS NA AMAZÔNIA OCIDENTAL
}

\author{
Julio Veras de Almeida e Silva ${ }^{1}$ \\ Fábio Augusto Gomes ${ }^{2}$ \\ Henrique Jorge de Freitas ${ }^{3}$ \\ Maria de Jesus Souza da Silva Barbosa ${ }^{4}$ \\ Jean Carlos Torres da Silva ${ }^{5}$
}

\begin{abstract}
SILVA, J. V. de A.; GOMES, F. A.; FREITAS, H. J. de; BARBOSA, M. de J. S. da S.; SILVA, J. C. T. da. Níveis de inclusão da torta da semente de cupuaçu na dieta de frangos caipiras criados na Amazônia Ocidental. Arq. Ciênc. Vet. Zool. UNIPAR, Umuarama, v. 20, n. 1, p. 1-7, jan./mar. 2017.
\end{abstract}

RESUMO: O experimento foi realizado com o intuito de avaliar os efeitos da inclusão da torta da semente de cupuaçu (TSC) nas dietas de frangos de linhagem caipira. O delineamento foi o inteiramente casualizado e foram utilizados 300 pintainhos de corte, sendo que os níveis de inclusão foram de $0,5,10,15$ e 20\%, de TSC nas rações. Cada tratamento possuía seis repetições com 10 aves. Foram analisados os níveis de inclusões da TSC nos períodos de 1-14, 1-28, 1-42, 1-56 e 1-70 dias de criação, em relação ao desempenho zootécnico (consumo de ração, peso vivo, conversão alimentar, eficiência alimentar e viabilidade dos frangos). Foi analisado também o rendimento de carcaça e a margem bruta relativa (MBR). A utilização da TSC na dieta dos frangos reduziu o consumo de ração e, consequentemente, o peso vivo, piorando a conversão alimentar, reduzindo a eficiência alimentar da ração e a viabilidade dos frangos. Não houve influência da inclusão da TSC sobre o rendimento de carcaça de machos, porém houve aumento no rendimento de moela vazia, intestinos e redução da gordura abdominal. Houve também redução na MBR, conforme os níveis crescentes de inclusão da TSC.

PALAVRAS-CHAVE: Alimentos alternativos. Criação de aves. Subprodutos.

\section{LEVELS OF CUPUAÇU SEED BY-PRODUCT IN FEEDSTUFF FOR FREE-RANGE BROILERS REARED IN THE WESTERN AMAZON}

\begin{abstract}
The experiment aimed to evaluate the effects of including the by-product of cupuacu seeds (BCS) in dietary feed of free-range broilers. A completely randomized design was used with 300 broiler chickens, and the inclusion levels ranged from $0,5,10,15$ to $20 \%$ of BCS in the feeds. Each treatment had six replicates with 10 birds. The BCS inclusion levels were analyzed in the total period from 1-14, 1-28, 1-42, 1-56, and 1-70 days old, in relation to the production performance (feed intake, live weight, feed conversion, feed efficiency and viability of broilers). The carcass yield and gross margin ratio (GMR) were also analyzed. The use of BCS in the feed of broiler chickens reduced the feed intake and consequently the live weight, worsening the feed conversion, and also reduced feed efficiency and the viability of broiler chickens. There was no influence of the inclusion levels of BCS on the carcass yield of males; nevertheless, there was an increase in the yield of intestines and gizzard, and a reduction of abdominal fat. There was also a reduction in the GMR as the BCS levels increased. KEY WORDS: Alternative foods. Broiler chicken. By-products.
\end{abstract}

\section{NIVELES DE INCLUSIÓN DEL SUBPRODUCTO DE SEMILLA DE CUPUAÇU EN LA DIETA DE POLLOS CAMPESINOS CREADOS EN LA AMAZÓNIA OCIDENTAL}

RESUMEN: El experimento fue realizado con el objetivo de evaluar los efectos de la inclusión del subproducto de semilla de cupuaçu (SSC) en las dietas de pollos de linaje campesino. El delineamiento fue enteramente casual y fueron utilizados 300 pollitos, siendo que los niveles de inclusión fueron de 0,5, 10, 15 y 20\% de SSC en las raciones. Cada tratamiento poseía seis repeticiones con 10 aves. Se analizó los niveles de inclusiones del SSC en los períodos de 1-14, 1-28, 1-42, 1-56 y 1-70 días de creación, en relación al desempeño zootécnico (consumo de ración, peso vivo, conversión alimentar, eficiencia alimentar y viabilidad de los pollos). Se ha analizado también el rendimiento del esqueleto y la margen bruta relativa (MBR). La utilización del SSC en la dieta de los pollos acortó el consumo de ración y consecuentemente el peso vivo, afectando de forma negativa la conversión alimentar, reduciendo la eficiencia alimentar de la ración y la viabilidad de los pollos. No hubo influencia de la inclusión del SSC sobre el rendimiento del esqueleto de machos, pero hubo aumento en el rendimiento de

DOI: https://doi.org/10.25110/arqvet.v20i1.2017.6312

${ }^{1}$ Eng. Agrônomo, Doutorando do Programa de Pós-Graduação em Sanidade e Produção Animal Sustentável na Amazônia Ocidental - Universidade Federal do Acre - UFAC, BR 364, Km 04, Distrito Industrial, CEP: 69919-673. julio-verass@hotmail.com

${ }^{2}$ Prof. Dr. do Programa de Pós-Graduação em Sanidade e Produção Animal Sustentável na Amazônia Ocidental, Universidade Federal do Acre - UFAC, BR 364, Km 04, Distrito Industrial, CEP: 69920-900. augusto.ufac@gmail.com

${ }^{3}$ Prof. Dr. do Programa de Pós-Graduação em Sanidade e Produção Animal Sustentável na Amazônia Ocidental, Universidade Federal do Acre - UFAC, BR 364, Km 04, Distrito Industrial, CEP: 69920-900. henriqufac@ufac.br

${ }^{4}$ Eng. Agrônoma, Mestre em Ciência Animal, Ramal Santa Lúcia, nº 868, Amapá, CEP: 69906-642, maria.agronomia@hotmail.com

${ }^{5}$ Médico Veterinário, Mestre em Ciência Animal, João de Barro, n 98, Ouricuri, CEP: 69903-224, jeanctorres@yahoo.com.br 
molleja vacía, intestinos y reducción de grasa abdominal. Hubo también reducción en la MBR, conforme los niveles crecientes de inclusión del SSC.

PALABRAS CLAVE: Alimentos alternativos. Creación de aves. Subproductos..

\section{Introdução}

O setor avícola no Brasil tem demonstrado grande potencial produtivo, tanto no mercado interno, como na exportação, visto que as demandas estão sempre aumentando e a população vem exigindo sempre qualidade e quantidade.

No sistema produtivo, a análise econômica é um fator determinante na decisão pela utilização ou não de um ingrediente na alimentação de aves. A utilização de fontes alimentares alternativas em rações para frangos de corte visando minimizar o custo por unidade de ganho de peso permite abordar em pesquisas parâmetros zootécnicos e econômicos (FERNANDES, et al., 2012).

Segundo Nunes et al. (2013), pesquisas utilizando resíduos agroindustriais têm sido realizadas no intuito de determinar as melhores opções de utilização de alimentos alternativos energéticos, proteicos e minerálicos, os quais, além de propiciar bom desempenho produtivo dos animais, reduzem o custo de alimentação, resultando em maior lucratividade ao produtor.

De acordo com Carneiro et al. (2009), a utilização de alimentos alternativos na dieta animal tem como principais objetivos reduzir os custos e incrementar a produtividade da atividade, principalmente durante os períodos de aumento nos preços do milho.

Os produtores de frangos do Estado do Acre, geralmente sofrem com aumento no custo de produção causado pela importação de grãos de milho e soja, devido à elevada demanda pelos mesmos, assim, a utilização de alimentos alternativos pode suprir, parcialmente as necessidades nutricionais das aves e trazer economias principalmente para os pequenos produtores que são os mais afetados com os custos de fretes das commodities.

Dentre os resíduos da agroindústria, tem-se a torta da semente de cupuaçu (TSC), que é resultado final da retirada do óleo da semente de cupuaçu, sendo a semente resultado do beneficiamento do fruto do cupuaçu, na indústria de cosméticos, ou despolpamento na indústria de polpas. $\mathrm{O}$ fruto é proveniente do cupuaçuzeiro (Theobroma grandiflorum, Schum), espécie arbórea nativa da região amazônica (MOTA, et al., 2014).

Nesse contexto, o objetivo deste trabalho foi avaliar o desempenho zootécnico e rendimento de carcaça dos frangos do tipo caipira alimentados com diferentes níveis de inclusão da torta da semente de cupuaçu na ração e avaliar também a viabilidade econômica da utilização mesma, sendo o público alvo os pequenos produtores, devido à sazonalidade do alimento alternativo.

\section{Material e Métodos}

O presente experimento foi aprovado pelo Comitê de Ética no Uso de Animais (CEUA) da Universidade Federal do Acre (UFAC), processo número 23107014902/201444 e protocolo número 17/2014.

O experimento foi conduzido no Setor de Avicultura da UFAC, situado no município de Rio Branco - Acre,
Amazônia Ocidental. O período experimental compreendeu os meses de maio a julho do ano de 2015.

Foram adquiridos 300 pintainhos de um dia de idade, mistos, da linhagem Pesadão mesclado. Esses foram distribuídos em 30 boxes contendo 10 aves cada, sendo cinco machos e cinco fêmeas por boxe, onde tinham ração e água ad libitum, durante os 70 dias experimentais. A coleta de dados de peso vivo e consumo de ração, era realizada a cada 14 dias, calculando também a conversão alimentar dos frangos no respectivo período de criação.

Os tratamentos consistiam em níveis diferentes de inclusão da torta da semente de cupuaçu' ${ }^{1}$ (TSC), sendo T1 ( $0 \%$ de TSC), T2 (5\% de TSC), T3 (10\% de TSC), T4 (15\% de TSC) e T5 (20\% de TSC). As rações foram formuladas conforme exigências nutricionais da linhagem, a base de fubá de milho e farelo de soja, sendo a TSC incluída na ração em relação à retirada do fubá de milho.

A proteína bruta foi analisada no laboratório de análises químicas da UFAC, Campus Floresta, localizado no município de Cruzeiro do Sul. As demais análises foram realizadas em laboratório particular, localizado no município de Campinas - SP. Na Tabela 1, estão apresentados os dados referentes à composição bromatológica da TSC.

Tabela 1: Composição bromatológica da torta da semente de cupuaçu utilizada nas rações.

\begin{tabular}{lcc}
\hline \multicolumn{1}{c}{ Análises } & Unidade & Resultado \\
\hline Proteína bruta & $\%$ & 14 \\
Energia bruta & $\mathrm{kcal} / \mathrm{kg}$ & 4.695 \\
Extrato etéreo & $\%$ & 15,81 \\
Fibra bruta & $\%$ & 15,18 \\
Material mineral & $\%$ & 5,79 \\
\hline
\end{tabular}

$\mathrm{Na}$ Tabela 2, estão apresentadas as composições calculadas das rações, na fase inicial (de um a 30 dias de idade) e de crescimento (de 31 a 70 dias).

Tabela 2: Composição calculada das rações em cada fase de criação.

\begin{tabular}{lccccc}
\hline & \multicolumn{5}{c}{ Inicial } \\
\hline & T1 & T2 & T3 & T4 & T5 \\
\hline Proteína bruta & 19,94 & 19,94 & 19,94 & 19,94 & 19,94 \\
Extrato etéreo & 3,20 & 3,69 & 4,18 & 4,67 & 5,16 \\
Fibra bruta & 3,53 & 4,14 & 4,74 & 5,35 & 5,95 \\
Minerais & 4,27 & 4,47 & 4,67 & 4,87 & 5,06 \\
\hline \multicolumn{5}{c}{ Crescimento } \\
\hline Proteína bruta & 19,14 & 19,14 & 19,14 & 19,14 & 19,14 \\
Extrato etéreo & 3,40 & 3,89 & 4,38 & 4,87 & 5,36 \\
Fibra bruta & 3,13 & 3,74 & 4,34 & 4,95 & 5,55 \\
Minerais & 3,87 & 4,07 & 4,27 & 4,47 & 4,66 \\
\hline
\end{tabular}

${ }^{1}$ Adquirida no projeto Reflorestamento Econômico Consorciado e Adensado (RECA), BR-364, Km 1071, Distrito de Nova Califórnia - Porto Velho - RO. 
Ao final do período experimental, duas aves foram separadas para o abate, sendo um macho e uma fêmea, de cada unidade experimental. Essas foram identificadas e submetidas a um jejum alimentar por 12 horas. Logo após, os frangos foram pesados e abatidos. Para a determinação do rendimento de carcaça, foi considerado o peso da carcaça limpa (sem pés e cabeça) em relação ao peso vivo após jejum, os rendimentos de moela vazia, gordura abdominal e intestinos, foram feitos em relação ao peso da carcaça.

A determinação da viabilidade econômica da TSC foi baseada na MBR (margem bruta relativa), considerando os custos variáveis das rações, conforme a seguinte fórmula:

$$
\mathrm{MBR}=\frac{(\text { PFTRAT } \times \text { KGS })-(\text { CRTRAT } \times \text { RSTRAT })}{(\text { PFCONT } \times \text { KGS })-(\text { CRCONT } \times \text { RSCONT })}
$$

Sendo, $\mathrm{MBR}=$ Margem bruta relativa; PFTRAT $=$ Peso final do frango no nível de inclusão da TSC; KG\$ = Preço do quilograma de frango caipira; $\mathrm{CRTRAT}=$ Consumo de ração/ave no nível de inclusão da TSC; R \$TRAT = Preço do quilograma de ração do nível de inclusão; PFCONT = Peso final do frango na dieta controle; CRCONT $=$ Consumo de ração/ave na dieta controle; $\mathrm{R} \$ C O N T=$ Preço do quilograma de ração da dieta controle.

O delineamento foi o inteiramente casualizado (DIC) sendo cinco tratamentos e seis repetições, totalizando 30 unidades amostrais. A análise estatística para comparação dos tratamentos foi feita por meio de Análise de Variância seguida do pós-teste de Student Newman Keuls (SNK) e regressão polinomial, sendo utilizado o programa SISVAR (FERREIRA, 2010). Para todos os testes foi considerado nível de significância de 5\%.

\section{Resultados}

Na Tabela 3, estão apresentados os dados referentes ao consumo de ração, onde os tratamentos com a inclusão da torta da semente de cupuaçu (TSC) provocaram uma redução no consumo de ração. Os tratamentos com 10 e $15 \%$ de inclusão foram iguais $(\mathrm{P}>0,05)$ até os 42 dias de idade, sendo diferentes nos períodos seguintes. $\mathrm{O}$ tratamento com $20 \%$ de inclusão apresentou a menor quantidade de consumo de ração em relação aos demais, a partir de 28 dias de idade. Assim, a cada $5 \%$ de inclusão da TSC, houve uma redução de $0,579 \mathrm{Kg}(\mathrm{Y}=-0,1158 \mathrm{x}+7,4262)$, no consumo de ração dos frangos avaliados.

Tabela 3: Consumo de ração $(\mathrm{kg})$ de frangos de linhagem caipira alimentados com diferentes níveis de inclusão da torta da semente de cupuaçu, em cada período de criação.

\begin{tabular}{cccccc}
\hline \multirow{2}{*}{$\begin{array}{c}\text { Níveis } \\
\text { de } \\
\text { inclusão }\end{array}$} & $\mathbf{1 - 1 4}$ & $\mathbf{1 - 2 8}$ & $\mathbf{1 - 4 2}$ & $\mathbf{1 - 5 6}$ & $\mathbf{1 - 7 0}$ \\
\cline { 2 - 6 } & & & & & \\
\hline 0 & $0,370 \mathrm{a}$ & $1,327 \mathrm{a}$ & $2,894 \mathrm{a}$ & $4,995 \mathrm{a}$ & $7,445 \mathrm{a}$ \\
5 & $0,357 \mathrm{ab}$ & $1,204 \mathrm{~b}$ & $2,596 \mathrm{~b}$ & $4,574 \mathrm{~b}$ & $6,819 \mathrm{~b}$ \\
10 & $0,342 \mathrm{bc}$ & $1,059 \mathrm{c}$ & $2,420 \mathrm{bc}$ & $4,202 \mathrm{c}$ & $6,286 \mathrm{c}$ \\
15 & $0,334 \mathrm{bc}$ & $0,989 \mathrm{c}$ & $2,312 \mathrm{c}$ & $3,823 \mathrm{~d}$ & $5,664 \mathrm{~d}$ \\
20 & $0,328 \mathrm{c}$ & $0,905 \mathrm{~d}$ & $1,860 \mathrm{~d}$ & $3,365 \mathrm{e}$ & $5,128 \mathrm{e}$ \\
$\mathrm{CV}(\%)$ & 4,87 & 5,56 & 7,84 & 5,58 & 5,33 \\
\hline
\end{tabular}

Médias com letras diferentes, na coluna, diferem entre si pelo teste SNK $(\mathrm{P}<0,05)$.

Com relação ao peso vivo (Tabela 4), constatou-se que houve efeito negativo $(\mathrm{P}<0,05)$ dos tratamentos com inclusão de TSC, onde, a partir do incremento de 5\% de TSC na ração, observou-se a redução no peso vivo dos mesmos. Assim, cada 5\% de inclusão da TSC houve uma redução de $0,363 \mathrm{Kg}(\mathrm{Y}=-0,0726 \mathrm{x}+3,0466)$ no peso final dos frangos avaliados.

Tabela 4: Peso vivo (kg) de frangos de linhagem caipira alimentados com diferentes níveis de inclusão da torta da semente de cupuaçu, em cada período de criação.

\begin{tabular}{cccccc}
\hline \multirow{2}{*}{$\begin{array}{c}\text { Níveis } \\
\text { de }\end{array}$} & \multicolumn{5}{c}{ Período } \\
\cline { 2 - 6 } inclusão & $\mathbf{1 - 1 4}$ & $\mathbf{1 - 2 8}$ & $\mathbf{1 - 4 2}$ & $\mathbf{1 - 5 6}$ & $\mathbf{1 - 7 0}$ \\
\hline 0 & $0,235 \mathrm{a}$ & $0,731 \mathrm{a}$ & $1,457 \mathrm{a}$ & $2,284 \mathrm{a}$ & $3,030 \mathrm{a}$ \\
5 & $0,214 \mathrm{~b}$ & $0,644 \mathrm{~b}$ & $1,238 \mathrm{~b}$ & $2,031 \mathrm{~b}$ & $2,687 \mathrm{~b}$ \\
10 & $0,193 \mathrm{c}$ & $0,545 \mathrm{c}$ & $1,039 \mathrm{c}$ & $1,707 \mathrm{c}$ & $2,333 \mathrm{c}$ \\
15 & $0,174 \mathrm{~d}$ & $0,470 \mathrm{~d}$ & $0,906 \mathrm{~d}$ & $1,463 \mathrm{~d}$ & $1,991 \mathrm{~d}$ \\
20 & $0,165 \mathrm{~d}$ & $0,383 \mathrm{e}$ & $0,672 \mathrm{e}$ & $1,129 \mathrm{e}$ & $1,564 \mathrm{e}$ \\
$\mathrm{CV}(\%)$ & 5,32 & 5,95 & 4,76 & 4,30 & 5,19 \\
\hline
\end{tabular}

Médias com letras diferentes, na coluna, diferem entre si pelo teste SNK $(\mathrm{P}<0,05)$.

O nível de inclusão de $5 \%$, a partir de 28 dias de criação, não apresentou diferença significativa $(\mathrm{P}>0,05)$ para conversão alimentar (Tabela 5) quando comparado ao tratamento sem inclusão de TSC. O tratamento que apresentou pior conversão foi o de $20 \%$ de TSC a partir de 56 dias. Assim em cada 5\% de inclusão da TSC, houve piora de 0,197 $\mathrm{Kg} / \mathrm{Kg}(\mathrm{Y}=0,0394 \mathrm{x}+2,374)$ na conversão alimentar dos frangos avaliados.

Tabela 5: Conversão alimentar $(\mathrm{kg} / \mathrm{kg})$ de frangos de linhagem caipira alimentados com diferentes níveis de inclusão da torta da semente de cupuaçu, em cada período de criação.

\begin{tabular}{cccccc}
\hline Níveis $\begin{array}{c}\text { Período } \\
\text { de } \\
\text { inclusão }\end{array}$ & $\mathbf{1 - 1 4}$ & $\mathbf{1 - 2 8}$ & $\mathbf{1 - 4 2}$ & $\mathbf{1 - 5 6}$ & $\mathbf{1 - 7 0}$ \\
\cline { 2 - 6 } & & & & & \\
\hline 0 & $1,57 \mathrm{a}$ & $1,82 \mathrm{a}$ & $1,99 \mathrm{a}$ & $2,19 \mathrm{a}$ & $2,46 \mathrm{a}$ \\
5 & $1,67 \mathrm{~b}$ & $1,87 \mathrm{a}$ & $2,10 \mathrm{ab}$ & $2,25 \mathrm{a}$ & $2,54 \mathrm{ab}$ \\
10 & $1,77 \mathrm{c}$ & $1,95 \mathrm{ab}$ & $2,33 \mathrm{bc}$ & $2,46 \mathrm{~b}$ & $2,70 \mathrm{bc}$ \\
15 & $1,93 \mathrm{~d}$ & $2,12 \mathrm{~b}$ & $2,55 \mathrm{~cd}$ & $2,62 \mathrm{~b}$ & $2,85 \mathrm{c}$ \\
20 & $2,00 \mathrm{~d}$ & $2,37 \mathrm{c}$ & $2,77 \mathrm{~d}$ & $2,99 \mathrm{c}$ & $3,29 \mathrm{~d}$ \\
$\mathrm{CV}(\%)$ & 4,20 & 7,36 & 8,37 & 5,32 & 4,85 \\
\hline
\end{tabular}

Médias com letras diferentes, na coluna, diferem entre si pelo teste SNK $(\mathrm{P}<0,05)$.

Com relação à eficiência alimentar (Tabela 6), os tratamentos com 0 e $5 \%$ de inclusão da TSC a partir de 28 dias, não apresentaram diferença significativa $(\mathrm{P}>0,05)$. $\mathrm{O}$ tratamento com $20 \%$ de TSC apresentou a pior eficiência alimentar a partir de 56 dias. 
Tabela 6: Eficiência alimentar da ração de frangos de linhagem caipira alimentados com diferentes níveis de inclusão da torta da semente de cupuaçu, em cada período da criação.

\begin{tabular}{cccccc}
\hline \multirow{2}{*}{$\begin{array}{c}\text { Níveis } \\
\text { de }\end{array}$} & \multicolumn{5}{c}{ Período } \\
\cline { 2 - 6 } inclusão & $1-14$ & $1-28$ & $1-42$ & $1-56$ & $1-70$ \\
\hline 0 & $0,64 \mathrm{a}$ & $0,55 \mathrm{a}$ & $0,50 \mathrm{a}$ & $0,46 \mathrm{a}$ & $0,41 \mathrm{a}$ \\
5 & $0,60 \mathrm{~b}$ & $0,54 \mathrm{ab}$ & $0,48 \mathrm{a}$ & $0,45 \mathrm{a}$ & $0,40 \mathrm{a}$ \\
10 & $0,57 \mathrm{c}$ & $0,51 \mathrm{ab}$ & $0,43 \mathrm{~b}$ & $0,41 \mathrm{~b}$ & $0,37 \mathrm{~b}$ \\
15 & $0,52 \mathrm{~d}$ & $0,48 \mathrm{~b}$ & $0,40 \mathrm{bc}$ & $0,38 \mathrm{c}$ & $0,35 \mathrm{c}$ \\
20 & $0,50 \mathrm{~d}$ & $0,42 \mathrm{c}$ & $0,36 \mathrm{c}$ & $0,34 \mathrm{~d}$ & $0,31 \mathrm{~d}$ \\
$\mathrm{CV}(\%)$ & 3,97 & 7,57 & 7,02 & 5,03 & 3,88 \\
\hline
\end{tabular}

Médias com letras diferentes, na coluna, diferem entre si pelo teste $\operatorname{SNK}(0,05)$.

A viabilidade (Tabela 7) foi afetada, sendo que a partir de 56 dias de criação os tratamentos de 15 e $20 \%$ apresentaram reduções significativas em relação aos tratamentos com 0 e 5\% de inclusão de TSC.

Tabela 7: Níveis de inclusão de torta da semente de cupuaçu com relação à viabilidade (\%) de frangos de linhagem caipira, em cada período da criação.

\begin{tabular}{cccccc}
\hline Níveis $\begin{array}{c}\text { Período } \\
\text { delusão }\end{array}$ & $1-14$ & $1-28$ & $1-42$ & $1-56$ & $1-70$ \\
\cline { 2 - 6 } inclucec & $100 \mathrm{a}$ & $100 \mathrm{a}$ & $100 \mathrm{a}$ & $100 \mathrm{a}$ & $100 \mathrm{a}$ \\
0 & $100 \mathrm{a}$ & $98 \mathrm{a}$ & $98 \mathrm{a}$ & $98 \mathrm{a}$ & $98 \mathrm{a}$ \\
5 & $100 \mathrm{a}$ & $98 \mathrm{a}$ & $88 \mathrm{ab}$ & $87 \mathrm{ab}$ & $87 \mathrm{ab}$ \\
10 & $100 \mathrm{a}$ & $97 \mathrm{a}$ & $88 \mathrm{ab}$ & $82 \mathrm{~b}$ & $80 \mathrm{~b}$ \\
15 & $100 \mathrm{a}$ & $93 \mathrm{a}$ & $78 \mathrm{~b}$ & $78 \mathrm{~b}$ & $78 \mathrm{~b}$ \\
20 & 0,00 & 6,52 & 10,66 & 10,87 & 11,92 \\
$\mathrm{CV}(\%)$ &
\end{tabular}

Médias com letras diferentes, na coluna, diferem entre si pelo teste SNK $(0,05)$.

Não houve diferença significativa no rendimento de carcaça de machos (Tabela 8). Com relação ao rendimento de moela vazia, o tratamento que apresentou maior rendimento foi o com $15 \%$ de inclusão da TSC.

Tabela 8: Rendimento de carcaça (RC), moela vazia (MV), gordura abdominal (GA) e intestinos (IN), de frangos machos de linhagem caipira alimentados com diferentes níveis de inclusão de torta da semente de cupuaçu na ração.

\begin{tabular}{ccccc}
\hline Níveis $\begin{array}{c}\text { de } \\
\text { inclusão }\end{array}$ & RC & MV & GA & IN \\
\cline { 2 - 5 } & $74,76 \mathrm{a}$ & $1,272 \mathrm{c}$ & $2,099 \mathrm{a}$ & $2,878 \mathrm{~b}$ \\
0 & $74,60 \mathrm{a}$ & $1,441 \mathrm{bc}$ & $2,260 \mathrm{a}$ & $3,336 \mathrm{~b}$ \\
5 & $75,63 \mathrm{a}$ & $1,757 \mathrm{~b}$ & $1,448 \mathrm{~b}$ & $3,262 \mathrm{~b}$ \\
10 & $73,58 \mathrm{a}$ & $2,205 \mathrm{a}$ & $1,424 \mathrm{~b}$ & $3,491 \mathrm{~b}$ \\
15 & $73,21 \mathrm{a}$ & $1,805 \mathrm{~b}$ & $0,663 \mathrm{c}$ & $4,370 \mathrm{a}$ \\
20 & 2,42 & 14,90 & 22,30 & 16,27 \\
CV (\%) &
\end{tabular}

Médias com letras diferentes, na coluna, diferem entre si pelo teste SNK $(0,05)$
Observou-se comportamento quadrático no rendimento de moela vazia dos machos em função da inclusão da TSC na ração. O nível para o valor máximo de rendimento $(1,95 \%)$ foi estimado em 16,95\% de inclusão (Figura 1).

Figura 1: Efeito quadrático dos níveis de inclusão da torta da semente de cupuaçu em relação ao rendimento de moela vazia.

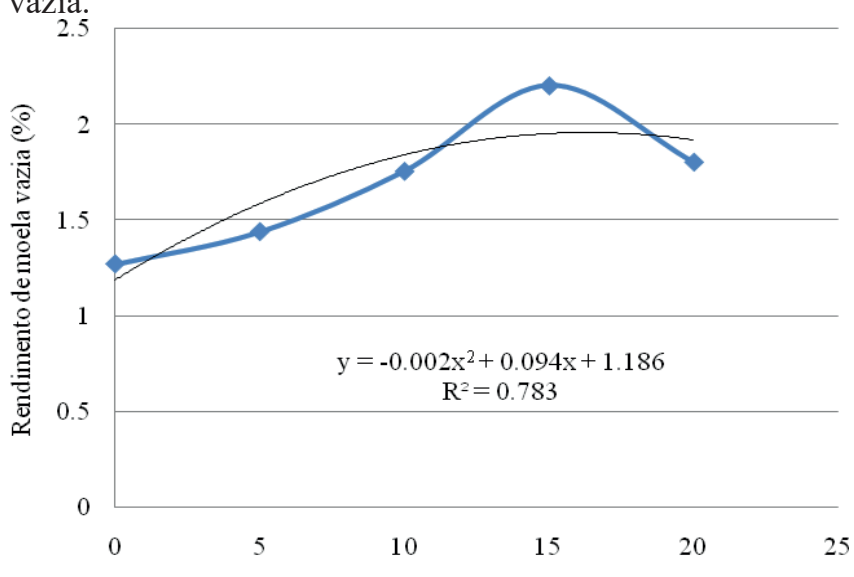

Níveis de inclusão da torta da semente de cupuaçu (\%)

Quanto à gordura abdominal, o tratamento com nível de inclusão de $20 \%$ apresentou menor quantidade da mesma diferindo $(\mathrm{P}<0,05)$ dos demais níveis de inclusão. Já os tratamentos com 0 e $5 \%$ apresentaram os maiores rendimentos de gordura abdominal.

Com relação ao rendimento de intestinos dos machos, houve diferença significativa $(\mathrm{P}<0,05)$ apenas no tratamento com $20 \%$ de inclusão da TSC, o mesmo apresentou o maior rendimento em relação aos demais.

No rendimento de carcaça das fêmeas (Tabela 9), o nível de $20 \%$, foi diferente $(\mathrm{P}>0,05)$ apenas dos tratamentos com 0 e $5 \%$ de inclusão da TSC, sendo inferior aos mesmos.

Tabela 9: Rendimento de carcaça (RC), moela vazia (MV), gordura abdominal (GA) e intestinos (IN), de frangos fêmeas de linhagem caipira alimentados com diferentes níveis de inclusão da torta da semente de cupuaçu na ração.

\begin{tabular}{ccccc}
\hline \multirow{2}{*}{$\begin{array}{c}\text { Níveis } \\
\text { de } \\
\text { inclusão }\end{array}$} & RC & MV & GA & IN \\
\cline { 2 - 5 } & $74,94 \mathrm{a}$ & $1,717 \mathrm{~b}$ & $3,058 \mathrm{a}$ & $3,033 \mathrm{~b}$ \\
0 & $74,78 \mathrm{a}$ & $1,681 \mathrm{~b}$ & $2,390 \mathrm{ab}$ & $3,025 \mathrm{~b}$ \\
5 & $74.20 \mathrm{ab}$ & $1,660 \mathrm{~b}$ & $2,580 \mathrm{ab}$ & $3,517 \mathrm{~b}$ \\
10 & $73,31 \mathrm{ab}$ & $2,083 \mathrm{a}$ & $2,000 \mathrm{ab}$ & $3,917 \mathrm{~b}$ \\
15 & $72,59 \mathrm{~b}$ & $1,935 \mathrm{ab}$ & $1,822 \mathrm{~b}$ & $4,914 \mathrm{a}$ \\
20 & 1,80 & 12,67 & 28,90 & 16,81 \\
$\mathrm{CV}(\%)$ &
\end{tabular}

Médias com letras diferentes, na coluna, diferem entre si pelo teste SNK $(0,05)$.

No rendimento de moela vazia das fêmeas, o tratamento com $15 \%$ de inclusão da TSC apresentou maior rendimento em relação aos tratamentos com 0,5 e $10 \%$ de TSC.

$\mathrm{O}$ rendimento de gordura abdominal apresentou-se diferente $(\mathrm{P}<0,05)$ somente em relação aos tratamentos com 0 e $20 \%$. Sendo que, o tratamento com $0 \%$ apresentou maior rendimento de gordura em relação ao tratamento com $20 \%$. 
Quanto ao rendimento de intestinos das fêmeas, o tratamento com $20 \%$ de inclusão da TSC apresentou o maior rendimento em relação aos outros tratamentos.

Os dados referentes à viabilidade econômica da utilização da TSC, referente a cada nível de inclusão, estão apresentados na Tabela 10, por meio da margem bruta relativa (MBR). Os custos referentes aos ingredientes da ração, bem como os preços pagos pelo quilograma do frango caipira, foram considerados de acordo com os valores praticados na região.

Tabela 10: Margem bruta relativa (MBR) dos tratamentos com inclusão da torta da semente de cupuaçu (TSC), consumo de ração/ave no nível de inclusão $\left(\mathrm{CR}_{\mathrm{TRAT}}\right)$ e preço do quilograma de ração no nível de inclusão $\left(\mathrm{R}_{\mathrm{TRAT}}\right)^{*}$.

\begin{tabular}{ccc}
\hline TSC (\%) & CRTRAT x R\$TRAT & MBR \\
\hline 5 & 6,889 & 89 \\
10 & 6,204 & 76 \\
15 & 5,449 & 65 \\
20 & 4,796 & 49 \\
\hline
\end{tabular}

A MB do tratamento controle foi 100. Os custos dos ingredientes utilizados foram de $0,72 \mathrm{R} \$ / \mathrm{Kg}$ do milho, $1,50 \mathrm{R} \$ / \mathrm{Kg}$ do concentrado e $0,1 \mathrm{R} \$ / \mathrm{Kg}$ da torta da semente de cupuaçu, o valor do frango foi de $14 \mathrm{R} \$ / \mathrm{Kg}$.

\section{Discussão}

Segundo Furtado et al. (2011), utilizando feno de maniçoba, que possui maior quantidade de fibra bruta comparada com a TSC, relataram que a quantidade de fibra, do alimento alternativo, pode ter reduzido o consumo de ração dos frangos, ocorrendo também aumento no rendimento de moela. Os autores ainda relatam que até o nível de 7,5\% de inclusão do feno, não houve diferença significativa $(\mathrm{P}>0,05)$ sobre o rendimento de carcaça dos frangos.

Em conclusão de seu estudo com farelo de coco, Bastos et al. (2007) afirmaram que quanto maior a fibra bruta da ração, maior é o volume seu, o que contribui para a redução do consumo devido à limitação na ingestão do alimento pelos frangos.

Segundo Carvalho et al. (2010), que utilizaram farelo de algodão na ração de frangos, apesar de possuir alta quantidade de fibra bruta, não houve diferença significativa $(\mathrm{P}>0,05)$ no consumo de ração. Os autores utilizaram uma ração isoaminoacídicas para metionina, metionina + cistina, lisina e treonina digestíveis.

O consumo de ração de aves está relacionado à busca de satisfazer, primariamente a sua necessidade energética. Assim, com o atendimento das necessidades de energia, a fome é saciada, controlando, portanto, o nível de consumo alimentar das aves (BERTECHINI, 2013). Como a torta da semente de cupuaçu (4695 kcal/kg) foi incluída na ração, proporcionalmente a retirada do milho $(3371 \mathrm{kcal} / \mathrm{kg})$, que possui menor energia, poderia então ter reduzido o consumo de ração pelas aves, à medida que foram aumentados os níveis de inclusão da TSC.

A redução no peso vivo pode ser atribuída à redução do consumo de ração, devido à inclusão da TSC. O tratamento que apresentou o pior peso vivo foi o de $20 \%$ de inclusão da TSC na ração. Costa et al. (2007), na avaliação do feno de maniçoba para frangos caipiras, relataram que o mesmo não pode ser incluído em níveis acima de $15 \%$. A fibra bruta do feno de maniçoba é maior que o valor da fibra bruta da TSC, sendo relatado que se for fornecido demasiadamente prejudica o desempenho produtivo dos frangos.

Segundo Togashi et al. (2008), não houve diferença significativa $(\mathrm{P}>0,05)$ no peso vivo de frangos (no nível de $8 \%$ de inclusão) alimentados com subprodutos da semente de maracujá, mesmo a semente contendo alta quantidade de fibra bruta $(31 \%$ e $3311 \mathrm{kcal} / \mathrm{kg}$, fibra e energia bruta, respectivamente), porém os autores não avaliaram níveis acima de $8 \%$, que poderiam talvez afetar o peso vivo dos frangos.

Segundo Parente et al. (2014), na utilização de resíduo da batata-doce em dietas para frangos de corte, observaram piora na conversão alimentar e no ganho de peso dos frangos. O subproduto possuía $4.499 \mathrm{Kcal} / \mathrm{Kg}$ de energia e 9,34\% de fibra bruta, que são valores próximos aos da composição da TSC.

Segundo Matos et al. (2015), em estudo com raspa de mandioca para frangos de corte, observaram-se que inclusões acima de $10 \%$ da mesma interferiram de forma negativa na eficiência alimentar da ração e consequentemente no peso corporal médio dos frangos avaliados. No presente trabalho, níveis de $10 \%$ a $20 \%$, apresentaram redução mais acentuada na eficiência alimentar, dos 42 dias até 70 dias de criação.

A viabilidade na criação de frangos pode ser afetada por razões sanitárias, metabólicas e de manejo. Como a variável avaliada no experimento era nutricional, possivelmente devido à baixa ingestão de nutrientes essenciais, os frangos ficaram mais susceptíveis a condições adversas, afetando na viabilidade dos mesmos, em níveis altos de TSC.

De acordo com Freitas et al. (2008) em estudo com farinha de varredura, com o aumento de seus níveis de inclusão não houve efeito sobre o rendimento de carcaça dos frangos, porém níveis crescentes da farinha, provocou a diminuição no consumo de ração e na atividade muscular da moela, diferente do observado com a TSC, onde houve redução no consumo de ração, porém, a moela se mostrou bem desenvolvida, com níveis altos da TSC.

Em experimento com farelo da raiz integral de mandioca na dieta de frangos caipiras, Carrijo et al. (2010), mostraram resultados semelhantes ao uso da TSC no nível de $20 \%$ de inclusão. A inclusão não afetou o rendimento de carcaça e com os acréscimos nos níveis do farelo, houve redução na quantidade de gordura abdominal dos frangos avaliados, porém sem prejudicar o desempenho produtivo dos frangos.

Em estudo referente ao efeito da energia sobre desempenho, rendimento de carcaça e gordura abdominal de frangos de corte, Barbosa et al. (2012) relataram que o aumento da energia na ração está diretamente ligado ao aumento de gordura abdominal, diferente do que sugere os resultados obtidos referente ao uso da TSC, onde o que realmente afetou o consumo e desempenho dos frangos não teria sido a energia da mesma, mas sim a sua quantidade de fibras, que possivelmente alterou o caráter concentrado da dieta, indisponibilizando potencialmente alguns nutrientes.

Em experimento utilizando polpa de caju na alimentação de frangos de corte, que possuía 12,1\% de Fibra bruta, $4.320 \mathrm{kcal} / \mathrm{kg}$ de energia e $14 \%$ de proteína bruta, Ramos et al. (2006) relataram que o alimento alternativo piorou a conversão alimentar, mas não afetou no rendimento de carcaça 
das aves, porém, a moela apresentou efeito linear positivo, para o acréscimo dos níveis de inclusão. Os autores comentaram que esses resultados aconteceram provavelmente devido à quantidade de fibra bruta que aumentou a atividade muscular da moela.

Em estudo com frangos de corte, utilizando dietas com diferentes níveis de fibras Pinheiro et al. (2008) concluíram que o aumento da fibra afetou de forma negativa a digestão ileal e total dos nutrientes pelos frangos, reduzindo o ganho de peso dos mesmos, havendo piora na conversão alimentar e aumento no peso dos cecos dos frangos. No presente experimento houve acréscimo também no peso dos intestinos no nível de $20 \%$ de inclusão da TSC, assim, a quantidade de fibra bruta pode ter causado este aumento.

Segundo Rungcharoen et al. (2013) avaliando o uso de subproduto de vermicelli tropical (resíduo de tipo de massa, na Tailândia), na alimentação de frangos de corte, recomendaram o nível máximo de $5 \%$ de inclusão nas dietas dos frangos, devido o aumento no rendimento da moela e do peso dos intestinos dos frangos que possuíram níveis acima de $5 \%$, sendo a quantidade de fibra bruta (32\%), a possível razão dos resultados obtidos.

No presente experimento os resultados podem ter sido afetados pela quantidade de fibra da TSC, que pode ter causado o aumento no tempo de retenção do alimento, provocando hipertrofia muscular da moela.

Com relação à margem bruta relativa (MBR), o valor da ração pela quantidade consumida do tratamento controle foi de 7,683 R\$, assim esse custo diminuiu conforme a inclusão da TSC, devido à redução no consumo de ração e valor da quantidade que foi consumida. Porém essa redução não refletiu na MBR, em função de que seu cálculo da mesma aborda também a produção por quilograma de ração consumida.

\section{Conclusão}

A utilização da torta da semente de cupuaçu prejudicou linearmente o desempenho dos frangos, não sendo opção viável na inclusão em rações para frangos de linhagem caipira.

\section{Referências}

BARBOSA, O. R. et al. Eficiência de sistemas evaporativos e dos níveis de energia na ração no desempenho de frangos de corte em crescimento. Semina: Ciências Agrárias, Londrina, v. 33, n. 4, p. 1589-1598, 2012.

BASTOS, S. C. et al. de P. Efeito da inclusão do farelo de coco em rações para frangos de corte. Revista Ciência Agronômica, Fortaleza, v. 38, n. 3, p. 297-303, 2007.

BERTECHINI, A. G. Metabolismo energético. In: Nutrição de monogástricos. 2.ed. Lavras: Ufla, 2013. cap. 7 , p. 118.

CARNEIRO, A. P. M. et al. Farelo de babaçu em rações para frangos de corte na fase final: desempenho, rendimento de carcaça e avaliação econômica. Ciência Animal Brasileira, Goiânia v.10, n.1, p. 40-47, 2009.
CARRIJO, A. S. et al. Níveis de farelo da raiz integral de mandioca em dietas para fêmeas de frangos caipiras. Revista Brasileira de Saúde e Produção Animal, Salvador, v. 11, n. 1, p. 131-139, 2010.

CARVALHO, C. B. et al. Avaliação nutricional do farelo de algodão de alta energia no desempenho produtivo e características de carcaças de frangos de corte. Ciência Rural, Santa Maria, v. 40, n. 5, p. 1166-1172, 2010.

COSTA, F. G. P. et al. Avaliação do feno de maniçoba (Manihot pseudoglaziovii Paz \& Hoffman) na alimentação de aves caipiras. Revista Caatinga, Mossoró, v. 20, n. 3, p. 42-48, 2007.

FERNANDES, R. T. V. et al. Aspectos gerais sobre alimentos alternativos na nutrição de aves. Revista Verde de Agroecologia e Desenvolvimento Sustentável, Mossoró, v. 7, n. 5, p. 66-72, 2012.

FERREIRA, D. F. Sisvar: sitema de análise de variância. Lavras: Ufla, 2010.

FREITAS, C. R. G. et al. Inclusão da farinha de varredura de mandioca em rações de frangos de corte. Acta Scientiarum. Animal Sciences, Maringá, v. 30, n. 2, p. 155-163, 2008.

FURTADO, D. A. et al. Desempenho de frangos alimentados com feno de maniçoba no semiárido paraibano. Revista Brasileira de Ciências Agrárias, Recife, v. 6, n. 4, p. 722-728, 2011.

MATOS, D. A. M. et al. Desempenho de frangos de corte na fase de 1 a 42 dias de idade alimentados com dietas contendo raspa de mandioca residual desidratada. In: CONGRESSO BRASILEIRO DE ZOOTECNIA, 25., 2015, Anais... Fortaleza: Associação Brasileira de Zootecnistas, 2015.

MOTA, D. A. et al. Torta de cupuaçu na alimentação de tourinhos nelore confinados, Boletim de Indústria Animal, Nova Odessa, v. 71, n. 4, , p. 309-316, 2014.

NUNES, J. K. et al. Alimentos alternativos ao milho na dieta de aves. Revista Eletrônica Nutritime, Viçosa, v. 10, n. 4, p. 2627-2645, 2013.

PARENTE, I. P. et al. Características nutricionais e utilização do resíduo de batata-doce em dietas de frangos de crescimento lento. Revista Brasileira de Saúde e Produção Animal, Salvador, v. 15, n. 2, p. 470-483, 2014.

PINHEIRO, C. C. et al. Digestibilidade dos nutrientes e desempenho de frangos de corte consumindo dietas formuladas com diferentes níveis de fibra e suplementadas com enzimas exógenas. Ciência Animal Brasileira, Jaboticabal, v. 9, n. 4, p. 984-996, 2008.

RAMOS, L. de S.N. et al. Polpa de caju em rações para frangos de corte na fase final: desempenho e características 
de carcaça. Revista Brasileira de Zootecnia, Piracicaba, v. 35, n. 3, p. 804-810, 2006.

RUNGCHAROEN, P. et al. By-product of tropical vermicelli waste as a novel alternative feedstuff in broiler diets.

Asian Australasian Journal of Animal Sciences, Nakhon Pathom, v. 26, n. 12, p. 1732-1741, 2013.

TOGASHI, C. K. et al. Subprodutos do maracujá em dietas para frangos de corte. Acta Scientiarum. Animal Sciences, Maringá, v. 30, n. 4, p. 395-400, 2008.

Recebido em: 28.04.2017 Aceito em: 30.05 .2017 